\title{
The preoperative glomerular filtration rate predicts new-onset postoperative atrial fibrillation in patients with hypertrophic obstructive cardiomyopathy who undergo isolated septal myectomy
}

\author{
Yanhai Meng ${ }^{1}$, Shuiyun Wang ${ }^{2}$, Ping Liu ${ }^{1}$, Yanbo Zhang ${ }^{1}$, Bing Tang ${ }^{2}$, Changsheng Zhu ${ }^{2}$, \\ Shengwei Wang ${ }^{2}$, Qiulan Yang ${ }^{1}$, Tao $\mathrm{Lu}^{2}$, Changrong $\mathrm{Nie}^{2}$ \\ ${ }^{1}$ Adult Surgery ICU, Department of Cardiovascular Surgery, State Key Laboratory of Cardiovascular Disease, Fuwai Hospital, National Center \\ for Cardiovascular Diseases, Chinese Academy of Medical Sciences and Peking Union Medical College, Beijing, China; ${ }^{2}$ Adult Surgery Center, \\ Department of Cardiovascular Surgery, State Key Laboratory of Cardiovascular Disease, Fuwai Hospital, National Center for Cardiovascular \\ Diseases, Chinese Academy of Medical Sciences and Peking Union Medical College, Beijing, China \\ Contributions: (I) Conception and design: Y Meng, S Wang; (II) Administrative support: S Wang, P Liu; (III) Provision of study materials or patients: \\ S Wang, P Liu, Y Zhang, C Zhu, S Wang; (IV) Collection and assembly of data: Y Meng, B Tang, C Zhu, Q Yang, T Lu, C Nie; (V) Data analysis \\ and interpretation: Y Meng, S Wang, P Liu, Y Zhang; (VI) Manuscript writing: All authors; (VII) Final approval of manuscript: All authors. \\ Correspondence to: Shuiyun Wang, MD. Fuwai Hospital, National Center for Cardiovascular Diseases, Chinese Academy of Medical Sciences and \\ Peking Union Medical College, Beijing 100037, China. Email: wsymd@sina.com.
}

Background: Few studies have focused on new-onset postoperative atrial fibrillation in patients with hypertrophic obstructive cardiomyopathy who have undergone septal myectomy. Therefore, we investigated the incidence and prognosis effects of postoperative atrial fibrillation following septal myectomy in patients with hypertensive obstructive cardiomyopathy. Additionally, we investigated the relationship of estimated glomerular filtration rate and postoperative atrial fibrillation.

Methods: Data from 300 patients with hypertrophic obstructive cardiomyopathy who underwent isolated surgical septal myectomy were collected from January 2012 to March 2018.

Results: The overall incidence of postoperative atrial fibrillation during hospitalization was $22.67 \%$ (68 of 300 patients). Patients with postoperative atrial fibrillation were older $(\mathrm{P}<0.001)$, had lower preoperative estimated glomerular filtration rate $(\mathrm{P}<0.001)$, and a larger preoperative left atrial diameter $(\mathrm{P}=0.038)$ compared to patients without. The preoperative estimated glomerular filtration rate predicted postoperative atrial fibrillation with sensitivity and specificity of 0.824 and $0.578(\mathrm{P}<0.001)$, respectively. Multivariate regression analyses showed that age [odds ratio $(\mathrm{OR})=1.090,95 \%$ confidence interval (CI): 1.034-1.110], an New York Heart Association functional class $\geq$ III (OR =2.985, 95\% CI: 1.349-6.604), hypertension ( $\mathrm{OR}=2.212$, 95\% CI: 1.062-4.608), a history of syncope (OR =3.890, 95\% CI: 1.741-8.692), and the preoperative estimated glomerular filtration rate $(\mathrm{OR}=0.981,95 \% \mathrm{CI}$ : 0.965-0.996) were independent risk factors associated in the development of postoperative atrial fibrillation. Survival analysis showed that the incidence of long-term cardiovascular events was higher in the patients with postoperative atrial fibrillation than that in the patients without the condition $(\mathrm{P}<0.001)$.

Conclusions: The preoperative estimated glomerular filtration rate was a moderate predictor of postoperative atrial fibrillation after septal myectomy. Postoperative atrial fibrillation affected the early recovery and the long-term prognoses of patients with hypertrophic obstructive cardiomyopathy who underwent septal myectomy.

Keywords: Hypertrophic cardiomyopathy; septal myectomy; atrial fibrillation (AF); predictors; prognosis 
Submitted Oct 28, 2020. Accepted for publication Jan 15, 2021.

doi: $10.21037 /$ jtd-20-3164

View this article at: http://dx.doi.org/10.21037/jtd-20-3164

\section{Introduction}

Postoperative atrial fibrillation (POAF) is a common complication after cardiac surgery, and it is associated with increased risks of stroke and mortality, prolonged hospital stays, and additional healthcare costs (1). The incidence of POAF following cardiac surgery is between $10 \%$ and $65 \%$ (2), which varies according to the type of surgery. POAF commonly occurs within 1 week of coronary artery bypass grafting (CABG) (3) and valve surgery (4). Different risk factors are associated with the development of new-onset POAF after cardiac surgery, including older age, left atrial (LA) enlargement, a history of congestive heart failure (CHF), obesity, and hypertension (5). However, most studies involving POAF investigated patients who have undergone $\mathrm{CABG}$ or valve surgery.

Hypertrophic obstructive cardiomyopathy (HOCM) is associated with diastolic dysfunction caused by left ventricular (LV) hypertrophy, reduced LV compliance, resulting in an increase in the $\mathrm{LV}$ end-diastolic pressure and the LA afterload (6). Septal myectomy is the gold standard treatment for HOCM that is refractory to medication (7). Atrial fibrillation (AF) is very common among patients with HOCM, and it underlies patients' worsening symptoms and quality of life (8).

Few studies have investigated the risk factors associated with POAF after septal myectomy despite its significant influence in the early recovery of patients with HOCM who have undergone septal myectomy. AF has a bidirectional relationship with renal dysfunction (9), which is a major risk factor for cardiovascular disease. Indeed, a decline in estimated glomerular filtration rate (eGFR) is associated with the development of AF (10), while the presence of $\mathrm{AF}$ promotes impaired renal function (11). Furthermore, declining eGFRs are associated with an increased risk of AF recurrence after successful electrical cardioversion (12). However, the association between renal dysfunction and POAF after cardiac surgery (13) is poorly described, especially among patients with HOCM. Therefore, this study aimed to investigate the incidence and prognostic effects of POAF following septal myectomy in patients with HOCM. Additionally, we evaluated the effects of renal dysfunction and other factors in the development of POAF.
We present the following article in accordance with the STORBE reporting checklist (available at http://dx.doi. org/10.21037/jtd-20-3164).

\section{Methods}

\section{Patient population}

Initially, we considered 698 consecutive patients who underwent septal myectomy at our institution between January 2012 and March 2018. Among them, 300 consecutive patients who underwent isolated septal myectomy were included in this study. HOCM was diagnosed based on the presence of LV hypertrophy in the absence of other cardiac or systemic diseases. Septal myectomies were performed on patients whose symptoms were refractory to treatment with drugs and whose maximum LV outflow tract gradients (LVOTGs) or midventricular gradients were $\geq 50 \mathrm{mmHg}$ at rest or following physiologic provocation. The criteria used to diagnose HOCM and its surgical indications were consistent with the 2011 American Heart Association/American College of Cardiology Foundation guidelines (14) and the 2014 European Society of Cardiology guidelines (15). This study's inclusion process and exclusion criteria are presented in Figure 1. The patients' demographic and clinical data, echocardiographic findings, and complete medical histories were collected prospectively, and their follow-up data were obtained from clinics or through telephone conversations between the patients and a blinded investigator. The study was conducted in accordance with the Declaration of Helsinki (as revised in 2013). The study was approved by ethics board of Fuwai Hospital (NO.: 20201315) and individual consent for this retrospective analysis was waived.

\section{Laboratory tests and echocardiography}

Venous blood samples were obtained during a patient's first admission before cardiac surgery. The plasma was isolated after centrifugation, and the serum creatinine $(\mathrm{sCr})$ and high-sensitivity C-reactive protein (hs-CRP) levels were measured. The patients' preoperative eGFRs were calculated using the Modification of Diet in Renal Disease (MDRD) 


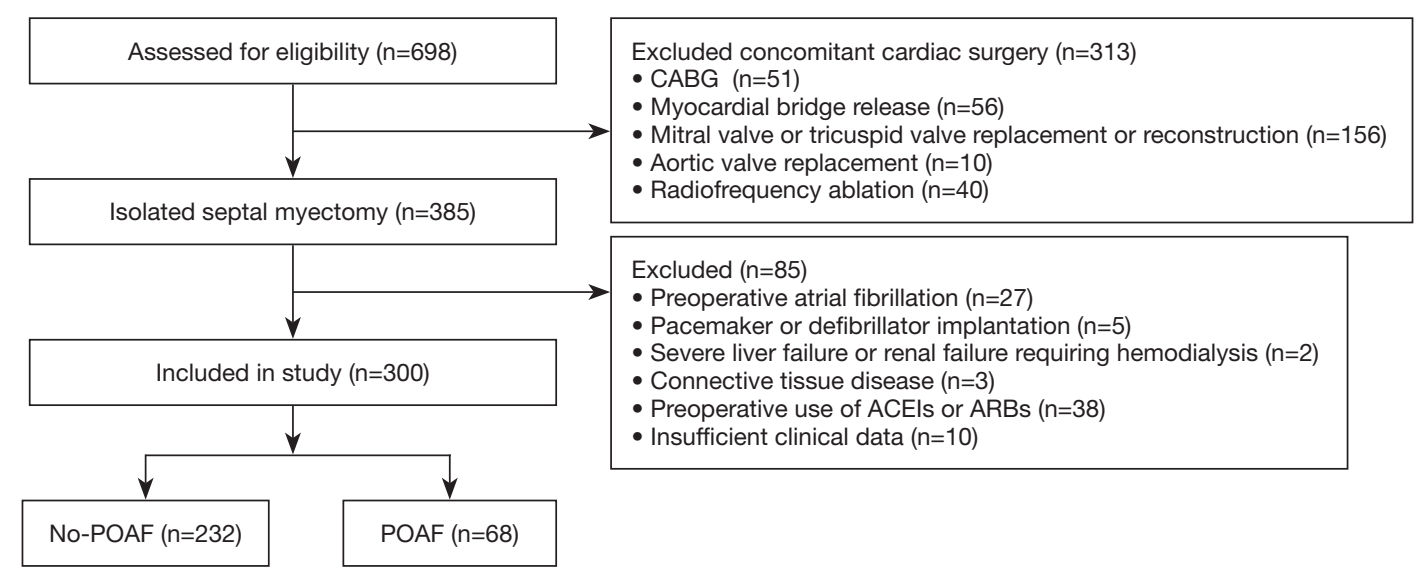

Figure 1 Flowchart of the study population selection process. POAF, postoperative atrial fibrillation; ACEIs, angiotensin-converting enzyme inhibitors; ARBs, angiotensin-receptor blockers.

Study equation that has been adapted for the Chinese population as follows (16): eGFR $\left(\mathrm{mL} / \mathrm{min} / 1.73 \mathrm{~m}^{2}\right)=175 \times$ $\mathrm{sCr}-1.234(\mathrm{mg} / \mathrm{dL}) \times$ age $-0.179(\times 0.79$, if female $)$.

Experienced physicians conducted the echocardiographic examinations using an E9 ultrasound system (General Electric Company, Boston, MA, USA). All patients underwent pre- and post-operative two-dimensional and Doppler echocardiography. The diameters of the cardiac chambers were recorded as the maximum anteroposterior diameters during the cardiac cycle. The thickness of the interventricular septum (IVS) was measured during diastole. The LVOTG was calculated using the simplified Bernoulli equation. The LV ejection fraction (LVEF) was measured according to the American Society of Echocardiography's recommendations. The methods are described in more detail in our previous publication (17).

\section{Cardiac surgery}

We performed a septal myectomy (extended Morrow procedure), as described previously (17). The hypertrophic ventricular septum that caused the systolic anterior motion of the mitral valve and LV outflow tract (LVOT) obstruction was resected. The resection was made along a longitudinal axis from approximately $4 \mathrm{~mm}$ below the aortic ring to the apex of the left ventricle and beyond the bases of the papillary muscles along the short axis, the myectomy began rightward to the nadir of the right aortic cusp, and it advanced to the left and terminated near the mitral anterior commissure. The hypertrophy of the LV anterior free wall that caused LVOT narrowing also required resection.
Furthermore, the anomalous chordal attachments affecting the LVOT were excised. Reoperation was required if intraoperative transesophageal echocardiography detected a postoperative LVOT gradient $>30 \mathrm{mmHg}$ or if the presence of more than moderate mitral valve regurgitation after weaning from cardiopulmonary bypass (CPB) is detected.

\section{POAF and outcome assessments}

Electrocardiography (ECG) and/or Holter monitoring assessed the patients postoperatively. All patients were monitored for the occurrence of POAF during their postoperative hospital stays using continuous electrocardiographic telemetry. Standard 12-lead electrocardiograms were recorded daily to inform staff of suspected arrhythmic events. POAF was defined as any sustained $\mathrm{AF}$ episode lasting $\geq 30$ min that was recorded by continuous telemetry during a patient's hospitalization or the need for treatment. A POAF diagnosis was confirmed by experienced cardiologists in ECG room.

\section{Postoperative medication}

For patients with symptomatic obstructive HOCM, betablockers were the first drug of choice; hence, beta-blockers were restarted as soon as possible during the postoperative period, unless they were contraindicated. Intravenous amiodarone or ibutilide was used if POAF occurred, and most POAF resolved spontaneously; these patients received beta-blockers continuously. Some patients presented with recurrent POAF episodes after the initial intervention, and they were prescribed with amiodarone. Anticoagulants, 
including warfarin or novel oral anticoagulants, continued for 3 months after discharge. All patients were followedup closely, and their treatments were adjusted promptly according to their most recent evaluations.

\section{Follow-up and study outcomes}

Information about a patient's clinical status after the myectomy was obtained through telephone conversations with the patient or members of their family. The final follow-up assessment was conducted in May 2019. The study's primary outcome was the development of new-onset POAF during hospitalization. Major adverse cardiac events were defined as a thromboembolic event, including an ischemic stroke, a transient ischemic attack, or a peripheral embolism, AF requiring hospitalization, $\mathrm{CHF}$ requiring hospitalization, and cardiovascular death.

\section{Statistical analyses}

The statistical analyses were performed using $\mathrm{IBM}^{\circledR} \mathrm{SPSS}^{\circledR}$, version 22.0 (IBM Corporation, Armonk, NY, USA). The continuous data are expressed as mean and standard deviation, and were compared using Student's $t$-test, while the continuous data are expressed as median (interquartile ranges), and were compared using the Mann-Whitney $\mathrm{U}$ test. The categorical data are presented as numbers (percentages), and they were compared using the chisquared test. The preoperative eGFR cutoff value was determined from the area under the receiver operating characteristic (ROC) curve. Correlation analyses were performed using Spearman's correlation coefficient. Univariate and backward stepwise multivariate logistic regression analyses were conducted to determine predictors of POAF. The nonparametric variables were log transformed. Variables with values of $\mathrm{P}<0.1$ in the univariate analysis were included in the multivariate logistic regression model. The Kaplan-Meier method was used with log-rank tests to evaluate event-free survival. A two-sided value of $\mathrm{P}<0.05$ was considered statistically significant.

\section{Results}

\section{Patients' baseline characteristics and the POAF rate after isolated septal myectomy}

Three hundred consecutive patients were enrolled in our study, of whom, 197 were men (65.67\%) and 103 were women
(34.33\%). The patients' mean age was $43.54 \pm 13.81$ years. POAF occurred in 68 of 300 patients $(22.67 \%)$ after a median duration of 2 days (range, $0-7$ days) after septal myectomy. Table 1 presents the baseline clinical characteristics of the patients with and without POAF. Compared to patients without $\mathrm{POAF}$, patients with $\mathrm{POAF}$ were significantly older $(41.12 \pm 13.47$ years vs. $51.81 \pm 11.64$ years, $\mathrm{P}<0.001)$, had higher mean body mass index $(\mathrm{BMI})$ $\left(24.75 \pm 3.81\right.$ vs. $\left.25.72 \pm 2.79 \mathrm{~kg} / \mathrm{m}^{2}, \mathrm{P}=0.023\right)$, and a higher New York Heart Association (NYHA) functional class (2.69 \pm 0.46 vs. $2.88 \pm 0.37, \mathrm{P}<0.001)$. Additionally, patients with POAF were more likely to have chest pain $(23.28 \%$ vs. $35.29 \%, \mathrm{P}=0.047)$, syncope $(9.91 \%$ vs. $23.53 \%$, $\mathrm{P}=0.002)$, and hypertension ( $13.36 \%$ vs. $29.41 \%, \mathrm{P}=0.002$ ). Additionally, patients with POAF had significantly lower preoperative eGFR compared to patients without POAF $\left(93.63 \pm 18.62\right.$ vs. $104.31 \pm 21.84 \mathrm{~mL} / \mathrm{min} / 1.73 \mathrm{~m}^{2}$, $\mathrm{P}<0.001)$. However, no significant difference was found in patients' preoperative sCr levels $(77.59 \pm 15.34 \mathrm{vs}$. $76.60 \pm 21.43 \mu \mathrm{mol} / \mathrm{L}, \mathrm{P}=0.740)$. There were no differences between the groups regarding the other baseline characteristics.

Table 2 summarizes the patients' preoperative and postoperative echocardiographic data. The patients with POAF had larger LA diameters (LADs) preoperatively $(46.13 \pm 6.45$ vs. $44.13 \pm 7.12 \mathrm{~mm}, \mathrm{P}=0.038)$ and postoperatively $(41.04 \pm 6.08$ vs. $38.02 \pm 6.10 \mathrm{~mm}, \mathrm{P}<0.001)$, and larger LV end-diastolic diameters (LVEDDs) preoperatively $(43.56 \pm 4.32$ vs. $41.78 \pm 5.36 \mathrm{~mm}, \mathrm{P}=0.019)$ and postoperatively $(45.08 \pm 4.19$ vs. $44.27 \pm 5.07 \mathrm{~mm}$, $\mathrm{P}=0.038)$ than the patients without POAF. The patients with and without POAF did not differ regarding the other echocardiographic variables.

The patients with POAF spent more time on mechanical ventilation $(21.81 \pm 16.04$ vs. $17.38 \pm 9.34 \mathrm{~h}, \mathrm{P}=0.033)$, in the intensive care unit (ICU) $(58.07 \pm 38.69$ vs. $53.63 \pm 29.35 \mathrm{~h}$, $\mathrm{P}=0.038)$, and in the hospital after the operation $(10.22 \pm 6.77$ vs. $7.81 \pm 3.23$ days, $\mathrm{P}=0.006$ ) than the patients without POAF. The groups did not differ regarding the CPB time, time to aortic occlusion, prevalence of early death, and major complication rates (Table 3).

\section{Predictors of POAF}

Logistic regression analyses were conducted to identify predictors of POAF in the patients with HOCM who underwent septal myectomy (Table 4). The univariate logistic regression analysis showed that age [odds ratio 
Table 1 Baseline characteristics and preoperative clinical variables of patients with and without POAF

\begin{tabular}{|c|c|c|c|}
\hline Characteristic & No-POAF (n=232) & POAF $(n=68)$ & $P$ value \\
\hline Sex (male), n (\%) & $158(68.1)$ & $39(57.35)$ & 0.101 \\
\hline Average body mass index $\left(\mathrm{kg} / \mathrm{m}^{2}\right)$, mean $\pm \mathrm{SD}$ & $24.75 \pm 3.81$ & $25.72 \pm 2.79$ & 0.023 \\
\hline Average NYHA functional class, mean $\pm \mathrm{SD}$ & $2.69 \pm 0.46$ & $2.88 \pm 0.37$ & $<0.001$ \\
\hline \multicolumn{4}{|l|}{ Symptoms, n (\%) } \\
\hline Dyspnea & $205(88.36)$ & $59(86.76)$ & 0.722 \\
\hline Chest pain & $54(23.28)$ & $24(35.29)$ & 0.047 \\
\hline Syncope & $23(9.91)$ & $16(23.53)$ & 0.002 \\
\hline Hypertension & $31(13.36)$ & $20(29.41)$ & 0.002 \\
\hline Hyperlipidemia & $28(12.07)$ & $13(19.12)$ & 0.137 \\
\hline Diabetes mellitus & $4(1.72)$ & $1(1.47)$ & 1.000 \\
\hline Coronary heart disease & $10(4.31)$ & $4(5.88)$ & 0.831 \\
\hline Chronic obstructive pulmonary disease & $3(1.29)$ & $1(1.47)$ & 1.000 \\
\hline Pulmonary hypertension & $15(6.47)$ & $4(5.88)$ & 1.000 \\
\hline Family history of SCD & $31(13.36)$ & $10(14.71)$ & 0.777 \\
\hline \multicolumn{4}{|l|}{ Blood factor level, mean \pm SD } \\
\hline Beta-blockers & $203(87.50)$ & $61(89.71)$ & 0.623 \\
\hline Diuretic & $38(16.38)$ & $12(17.65)$ & 0.805 \\
\hline
\end{tabular}

POAF, postoperative atrial fibrillation; SD, standard deviation; NYHA, New York Heart Association; SCD, sudden cardiac death; eGFR, estimated glomerular filtration rate; hs-CRP, high sensitivity C-reactive protein.

$(\mathrm{OR})=1.072,95 \%$ confidence interval $(\mathrm{CI}): 1.045-1.100$, $\mathrm{P}<0.001]$, the preoperative LAD $(\mathrm{OR}=1.042,95 \% \mathrm{CI}$ : $1.002-1.084, \mathrm{P}=0.039)$, the LVEDD (OR $=1.067,95 \% \mathrm{CI}$ : $1.010-1.127, \mathrm{P}=0.020)$, hypertension $(\mathrm{OR}=2.702,95 \% \mathrm{CI}$ : $1.418-5.146, \mathrm{P}=0.003)$, a history of chest pain $(\mathrm{OR}=1.798$, 95\% CI: $1.004-3.221, \mathrm{P}=0.049$ ), a history of syncope (OR $=2.796,95 \% \mathrm{CI}: 1.379-5.668, \mathrm{P}=0.004)$, an NHYA functional class $\geq$ III (OR $=2.910,95 \%$ CI: $1.367-6.189$, $\mathrm{P}=0.006)$, and the preoperative eGFR $(\mathrm{OR}=0.975,95 \%$
CI: 0.961-0.989, $\mathrm{P}<0.001)$ were potential risk factors associated with POAF. The multivariate logistic regression analysis showed that age (OR $=1.090,95 \%$ CI: $1.034-1.110$, $\mathrm{P}<0.001)$, an NHYA functional class $\geq \mathrm{III}(\mathrm{OR}=2.985,95 \%$ CI: 1.349-6.604, $\mathrm{P}=0.007)$, hypertension ( $\mathrm{OR}=2.212$, 95\% CI: 1.062-4.608, $\mathrm{P}=0.034)$, a history of syncope $(\mathrm{OR}=3.890$, 95\% CI: $1.741-8.692, \mathrm{P}=0.001)$, and the preoperative eGFR (OR $=0.981,95 \%$ CI: $0.965-0.996, \mathrm{P}=0.015)$ were independent risk factors associated with POAF. 
Table 2 Pre- and post-operative echocardiography characteristics of patients with and without POAF

\begin{tabular}{|c|c|c|c|}
\hline Variables & No-POAF ( $n=232)$ & POAF $(n=68)$ & $P$ value \\
\hline Interventricular septum (mm) & $19.25 \pm 4.66$ & $18.40 \pm 3.88$ & 0.136 \\
\hline Left atrial diameter $(\mathrm{mm})$ & $44.13 \pm 7.12$ & $46.13 \pm 6.45$ & 0.038 \\
\hline Left ventricular end diastolic diameter (mm) & $41.78 \pm 5.36$ & $43.56 \pm 4.32$ & 0.019 \\
\hline Left ventricular outflow tract peak gradient $(\mathrm{mmHg})$ & $76.80 \pm 21.29$ & $77.99 \pm 15.34$ & 0.462 \\
\hline \multicolumn{4}{|l|}{ Post-operative variables, mean \pm SD } \\
\hline Interventricular septum (mm) & $14.41 \pm 3.96$ & $14.49 \pm 3.21$ & 0.892 \\
\hline Left atrial diameter $(\mathrm{mm})$ & $38.02 \pm 6.10$ & $41.04 \pm 6.08$ & $<0.001$ \\
\hline Left ventricular outflow tract peak gradient $(\mathrm{mmHg})$ & $11.23 \pm 7.96$ & $12.38 \pm 12.64$ & 0.367 \\
\hline
\end{tabular}

POAF, postoperative atrial fibrillation.

Table 3 Comparison of perioperative outcomes and early complications with and without POAF

\begin{tabular}{lcc}
\hline Variables & No-POAF $(n=232)$ & POAF $(n=68)$ \\
\hline Perioperative outcomes & & P value \\
Cardiopulmonary bypass time (min) & $96.80 \pm 22.96$ & $94.10 \pm 20.17$ \\
Aortic clamp time (min) & $63.77 \pm 16.04$ & $61.68 \pm 15.29$ \\
Ventilation time (hour) & $17.38 \pm 9.34$ & $21.81 \pm 16.04$ \\
ICU stay (hour) & $53.63 \pm 29.35$ & $58.07 \pm 38.69$ \\
Length of stay (day) & $7.81 \pm 3.23$ & $10.22 \pm 6.77$ \\
Average NYHA functional class & $1.16 \pm 0.45$ & $1.21 \pm 0.51$ \\
Early complications, $n$ (\%) & & 0.0384 \\
Perioperative death & $1(0.43)$ & 0.038 \\
Infection of incision & $2(0.86)$ & $1(1.47)$ \\
Reoperation for bleeding & $2(0.86)$ & $1(1.47)$ \\
Permanent pacemaker & $4(1.72)$ & 0.447 \\
\hline
\end{tabular}

ICU, intensive care unit; NYHA, New York Heart Association; POAF, postoperative atrial fibrillation.

\section{Preoperative eGFR and POAF prediction}

ROC curve analyses evaluated the effectiveness of preoperative eGFR in identifying patients with POAF (Figure 2). The area under the ROC curve for the preoperative eGFR was 0.731 (95\% CI: 0.677-0.780, $\mathrm{P}<0.001)$. The optimal preoperative eGFR cutoff value for the prediction of POAF in patients with HOCM who underwent septal myectomy was $103.23 \mathrm{~mL} / \mathrm{min} / 1.73 \mathrm{~m}^{2}$, which had a sensitivity of 0.824 and a specificity of 0.578 . A lower preoperative eGFR correlated negatively with the LAD $(r=-0.228, \mathrm{P}<0.001)$, the BMI $(\mathrm{r}=-0.301, \mathrm{P}<0.001)$, and $\mathrm{POAF}(\mathrm{r}=-0.335, \mathrm{P}<0.001)$. 
Table 4 Logistic analysis for predictors of postoperative atrial fibrillation

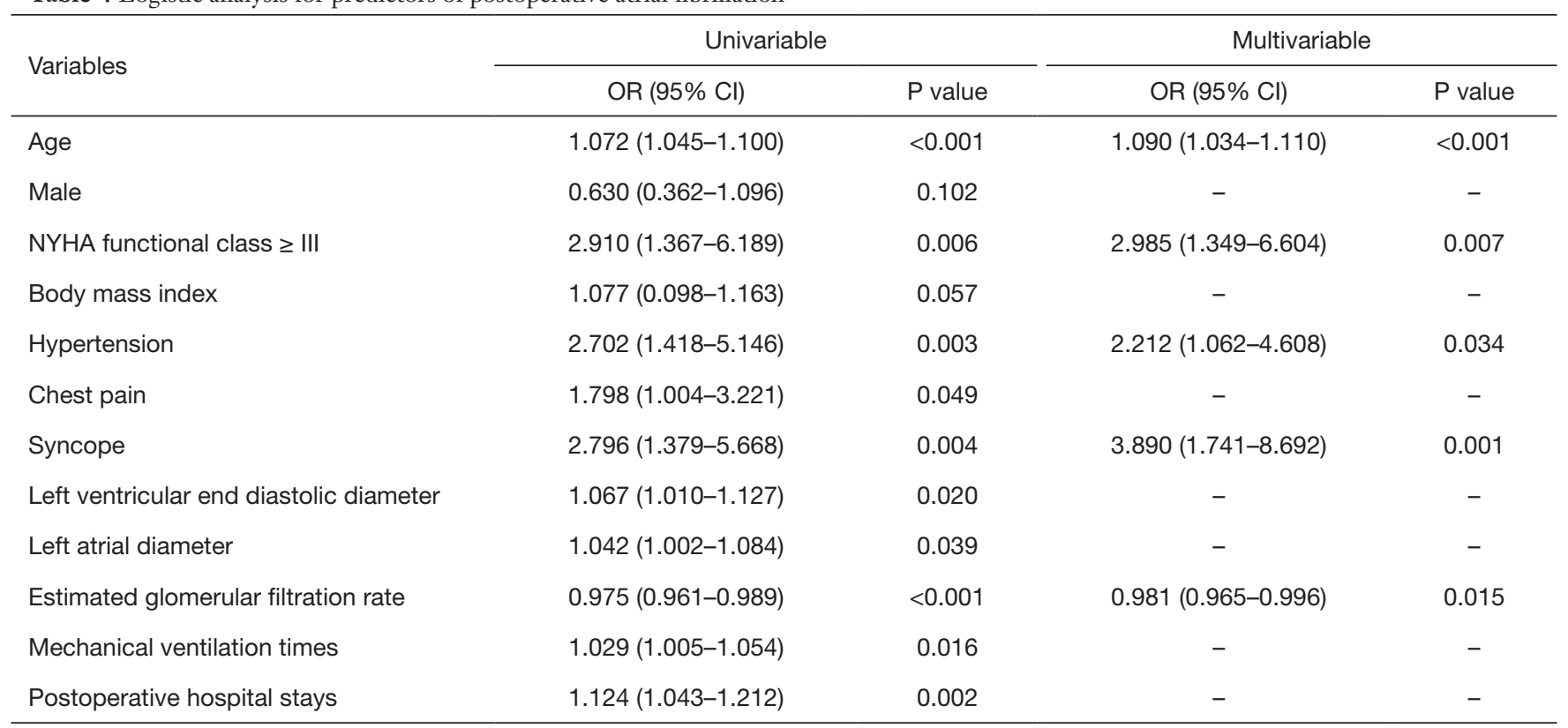

OR, odds ratio; $\mathrm{Cl}$, confidence interval; NYHA, New York Heart Association.

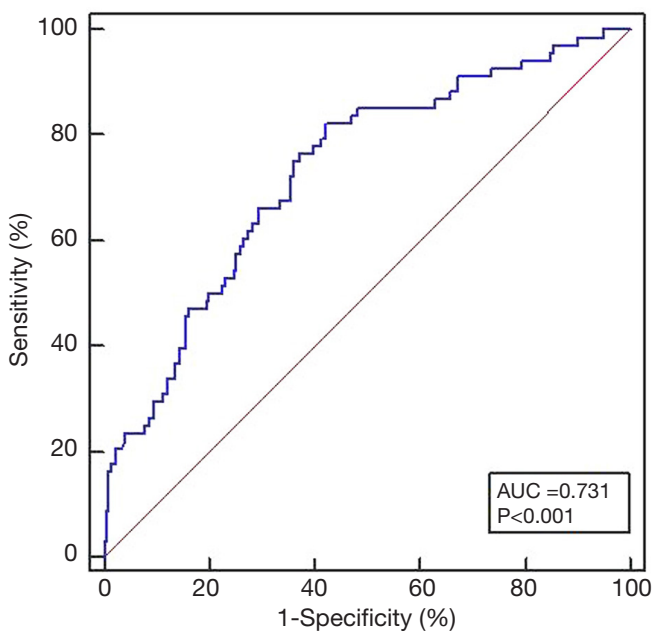

Figure 2 Receiver operating characteristic curves of estimated glomerular filtration rate level for predicting POAF after septal myectomy. The optimal cut-off point was $103.23 \mathrm{~mL} / \mathrm{min} / 1.73 \mathrm{~m}^{2}$ predicted AF after myectomy with sensitivity and specificity of $82.35 \%$ and $57.76 \%$ [area under the curve (AUC) 0.731 (95\% CI: 0.677-0.780), $\mathrm{P}<0.001]$. POAF, postoperative atrial fibrillation; AF, atrial fibrillation; CI, confidence interval.

\section{POAF and major adverse cardiac events after isolated septal myectomy}

All patients were followed up successfully, and none of the patients died during the early postoperative period. All patients who had minor complications were discharged uneventfully. The mean follow-up interval was $27.65 \pm 19.47$ months (range, 3-96 months). In total, 40 patients $(13.33 \%)$ experienced major adverse cardiac events, which included 4 sudden deaths, 2 tumorassociated deaths, 10 patients who were readmitted with AF, 6 patients who had permanent pacemakers implanted, 13 patients who were hospitalized for heart failure, and 5 patients who experienced stroke and developed thrombi (Table 5). Compared with the patients without POAF, those with POAF were more likely to be hospitalized for AF occurrence $(8.82 \%$ vs. $1.72 \%, \mathrm{P}=0.004)$, CHF $(10.29 \%$ vs. $2.59 \%, \mathrm{P}=0.006)$, and stroke and thrombi $(4.41 \%$ vs. $0.86 \%, \mathrm{P}=0.040)$. The Kaplan-Meier survival curves showed a lower event-free survival rate for the patients with POAF compared with patients without POAF $(\mathrm{P}=0.033$, Figure 3$)$. 
Table 5 Major adverse cardiovascular events during follow-up

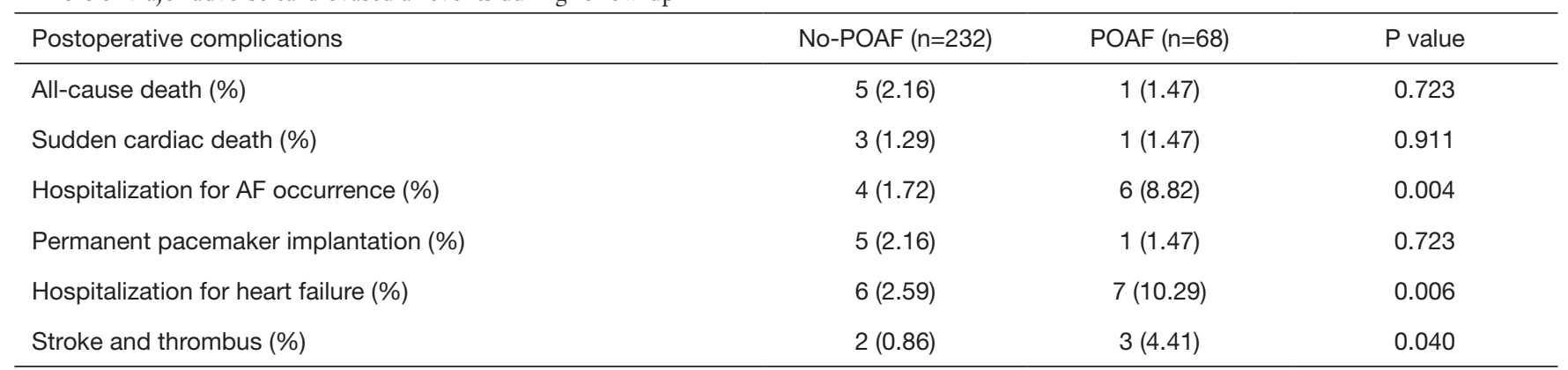

POAF, postoperative atrial fibrillation; AF, atrial fibrillation.

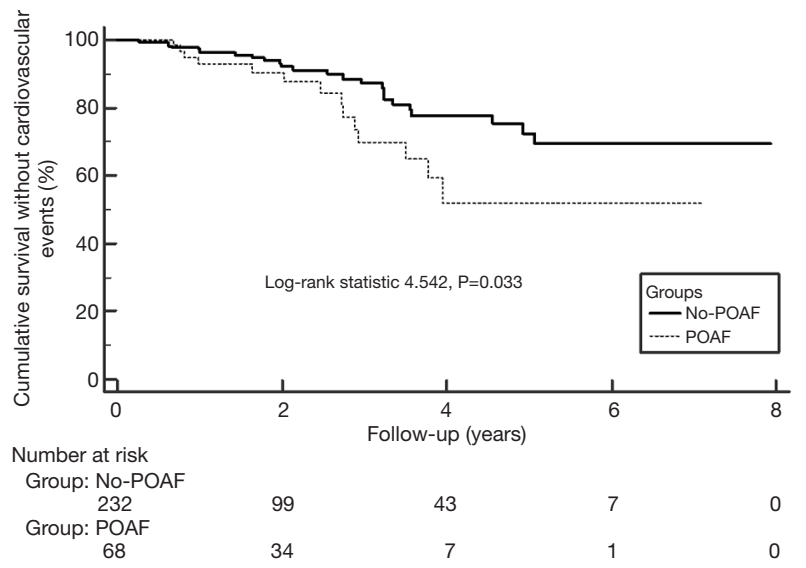

Figure 3 Event-free survival in patients with HOCM after myectomy according to the occurrence of POAF or not. Eventfree survival in patients after myectomy with POAF and without POAF was different (log-rank statistic 4.542, $\mathrm{P}=0.033$ ). POAF, postoperative atrial fibrillation; HOCM, hypertrophic obstructive cardiomyopathy.

\section{Discussion}

This study's findings demonstrated the incidence and the risk factors associated with POAF in patients with HOCM who had undergone isolated septal myectomy. Our findings showed that the preoperative eGFR had a moderate ability in predicting the occurrence of $\mathrm{POAF}$ in patients with HOCM. The patients with concurrent HOCM and POAF had a higher incidence of adverse cardiovascular events during follow-up, and their long-term event-free survival rate was lower compared to patients with HOCM alone. In addition to identifying risk factors that are well-established among patients who have undergone CABG or aortic valve replacement such as age and $\operatorname{LAD}(4,5)$, we identified previously unknown risk factors that were associated with POAF after septal myectomy, including the preoperative eGFR.

We excluded the influence of other procedures, including mitral valvuloplasty or replacement, and CABG, which can prolong the operative time (18) and CPB time (19), and increase the levels of inflammatory factors and myocardial injury, which may lead to AF. According to a study in our center (20), the incidence of POAF is low due to inconsistent criteria and time frame. In this study we collected consecutive cases of isolated septal myectomy, regardless of MRI results.

Our results showed that the patients with POAF were older, were more likely to have larger LADs, had higher BMIs, had a higher incidence of hypertension, and had worse NYHA functional classifications than the patients who did not have POAF, and, in general, these were independent risk factors associated with POAF after septal myectomy, which concurs with previous studies' findings $(4,5)$. Age is an independent risk factor for AF in the general population (21), and it is associated with atrial deterioration. Patients with HOCM who undergo septal myectomy are younger than patients who undergo CABG (22). However, the incidence of POAF following septal myectomy is not lower, which suggests that other factors may be involved in the occurrence of POAF after septal myectomy. Many studies have investigated the relationship between the LAD and $\mathrm{AF}(4,5,8)$, and recent studies' findings have shown that the effectiveness of the LA volume index and LA function in predicting $\mathrm{AF}$ are better than predictions based on two-dimensional LAD measurements (23). Diastolic dysfunction is closely related to patients' susceptibility to atrial arrhythmia (23-25), and it is clearly associated with a high prevalence of POAF (25). LV diastolic dysfunction is markedly more severe in patients with HOCM compared 
with the general population (7). Hypertension is the most significant risk factor associated with the development of $\mathrm{LV}$ diastolic dysfunction (26), and by aggravating $\mathrm{LV}$ diastolic dysfunction, it may be involved in the development of POAF. The preoperative LVEF did not differ between the groups of patients with HOCM in this study, because $\mathrm{LV}$ diastolic dysfunction is a key characteristic of these patients (15). However, our study's findings showed that the patients with POAF had high preoperative NYHA functional classifications, and that chest pain and syncope occurred more frequently and were more distinctive in the patients with POAF. Hence, the condition of patients with POAF is more serious, and their diastolic dysfunction and myocardial ischemia are more severe.

There were no differences between the groups regarding the $\mathrm{CPB}$ time, the aortic cross-clamp time, or the expression of hs-CRP, which may indicate the degree of inflammation; however, other studies' findings (19) are inconsistent in this regard. Some investigators $(19,27)$ believe that $\mathrm{CPB}$, inadequate atrial protection, a longer ischemic time, and increased levels of inflammatory factors cause POAF, but other studies' findings have not shown any clear correlations between these factors and the occurrence of POAF (28). We showed that POAF was an independent risk factor associated with prolonged ICU and hospital stays, which suggests that while POAF affects patients' early recoveries and, therefore, increases healthcare costs, it was not associated with higher early complication rates, which concurs with the conclusions from other studies $(29,30)$.

In this study, we found that the preoperative eGFR, rather than the $\mathrm{sCr}$ level, may be an independent risk factor for POAF in patients with HOCM who undergo septal myectomy, and that the preoperative eGFR may have a moderate ability to predict the occurrence of POAF; these findings are similar to those from studies of 676 patients who underwent CABG (31). The patients in the POAF group had a significantly lower preoperative eGFR and higher sCr level, but the trend relating to the preoperative eGFR was more distinct. Previous studies' findings have also shown that the eGFR, which is frequently monitored in clinical practice, describes renal dysfunction more accurately and rapidly (32). The mechanism underlying the occurrence of AF that results from renal insufficiency is not fully understood; it may be caused by hypertension, fluid overload, the pathological activation of the reninangiotensin-aldosterone system (RAAS), and the subsequent enhancement of myocardial fibrosis (33). Other studies' findings have shown a common pathophysiological process underlying an eGFR decline and the occurrence of AF (34). Watanabe et al. (9) undertook a community-based, prospective study, and reported a bidirectional risk between renal insufficiency and AF. The findings from a recent metaanalysis of patients who underwent catheter ablation have also indicated the presence of a bidirectional association between $\mathrm{AF}$ and renal function (35).

While elevated LV pressure, LA pressure, and LA enlargement in patients with HOCM increase their susceptibility to AF (36), widespread LV diastolic dysfunction in these patients can cause hemodynamic abnormalities and venous congestion, which activates the RAAS that can compromise renal function and promote the decline of the eGFR $(37,38)$. In this study, the preoperative eGFR cut-off value for the prediction of POAF was in the normal range, which might indicate a change in the renal function of HOCM patients with diastolic dysfunction, even if eGFR was in the low-normal level. We further found that a lower preoperative eGFR was significantly associated with a larger LAD and IVS, which suggests that the eGFR may reflect, in part, an LA enlargement and IVS thickening. LA enlargement is an important cause of AF, and the correlation between renal insufficiency and AF may be related to LA enlargement. For the general population, eGFR is within the normal range, while for the patients with HOCM, lower than the cut-off value may indicate the severity of $\mathrm{LV}$ diastolic dysfunction and the changes of tension and hormone secretion caused by LA enlargement, and then increases the susceptibility to POAF. Therefore, the preoperative eGFR may predict the occurrence POAF after septal myectomy.

Our results showed that a lower preoperative eGFR was significantly associated with a high incidence of new-onset POAF and a longer hospital stay. Therefore, preventing and reducing renal function deterioration and avoiding nephrotoxic drugs are important for patients with HOCM who are undergoing surgery. Treating common diseases such as hypertension can improve renal function and lead to favorable outcomes $(38,39)$. To stabilize the heart's rhythm, we continued to use beta-blockers and calcium channel blockers to avoid AF, and when AF occurred, drug conversion therapy, which comprised of amiodarone or ibutilide, was required. In this study, the incidence of thrombi and stroke was higher among the patients with POAF compared to patients without POAF. Additionally, the sex, age 65-74 years, age $\geq 75$ years, CHF, hypertension, diabetes mellitus, and stroke or transient ischemic attack or thromboembolism-vascular disease, cannot predict 
thrombotic events very reliably (40). Therefore, we consider that antithrombotic drugs should be used to prevent thrombi in patients with HOCM and POAF after septal myectomy.

\section{Limitations}

The present study analyzed data from a retrospective, observational cohort; therefore, its findings should be interpreted in the context of the limitations associated with retrospective analyses. First, there may have been biases regarding the selection and enrollment of the patients. To avoid bias, we excluded patients with a history of AF, which may have been associated with a high predictive value. Second, the observed relationship between POAF and the preoperative eGFR can only be considered an association and not a causal relationship. Third, we accounted for POAF that occurred in the hospital, and paroxysmal and asymptomatic AF after discharge were not considered; hence, the incidence of POAF may have been underestimated. However, a recent study's findings confirmed that most POAF occurs 2-5 days after cardiac surgery (2). Continuously monitoring heart rhythms during hospitalization captures most $\mathrm{AF}$, and the proportion of patients with POAF who are discharged from hospital is very small. Fourth, the findings from a recent study (41) show that administering beta-blockers preoperatively can reduce the incidence of POAF after cardiac surgery. In this study, most patients received beta-blockers before surgery, which may have led to an underestimation of the actual incidence of POAF. Finally, the MDRD study equation was used to calculate the eGFRs, which are affected by age, and age is a risk factor that is also associated with POAF; therefore, POAF may have occurred as a consequence of the patients' increasing age.

\section{Conclusions}

In conclusion, POAF is the most common arrhythmic condition that occurs after septal myectomy among patients with HOCM, and it significantly affects their early recovery and long-term prognoses. A lower preoperative eGFR correlated significantly with the risk and occurrence of POAF. The preoperative eGFR had a moderate ability to predict the occurrence of POAF. However, multicenter, prospective studies involving large numbers of patients are required to analyze and predict POAF among patients with HOCM after they have undergone septal myectomy.

\section{Acknowledgments}

We would like to thank Editage (www.editage.cn) for English language editing.

Funding: This work was supported by Capital's Funds for Health Improvement and Research (CFH 2020-2-4036), and clinical and translational medicine research fund of Chinese Academy of Medical Sciences (2019XK320054).

\section{Footnote}

Reporting Checklist: The authors have completed the STROBE reporting checklist. Available at http://dx.doi. org/10.21037/jtd-20-3164

Data Sharing Statement: Available at http://dx.doi. org/10.21037/jtd-20-3164

Conflicts of Interest: All authors have completed the ICMJE uniform disclosure form (available at http://dx.doi. org/10.21037/jtd-20-3164). The authors have no conflicts of interest to declare.

Ethical Statement: The authors are accountable for all aspects of the work in ensuring that questions related to the accuracy or integrity of any part of the work are appropriately investigated and resolved. The study was conducted in accordance with the Declaration of Helsinki (as revised in 2013). The study was approved by ethics board of Fuwai hospital (NO.: 20201315) and individual consent for this retrospective analysis was waived.

Open Access Statement: This is an Open Access article distributed in accordance with the Creative Commons Attribution-NonCommercial-NoDerivs 4.0 International License (CC BY-NC-ND 4.0), which permits the noncommercial replication and distribution of the article with the strict proviso that no changes or edits are made and the original work is properly cited (including links to both the formal publication through the relevant DOI and the license). See: https://creativecommons.org/licenses/by-nc-nd/4.0/.

\section{References}

1. El-Chami MF, Kilgo P, Thourani V, et al. New-onset atrial fibrillation predicts long-term mortality after coronary artery bypass graft. J Am Coll Cardiol 2010;55:1370-6.

2. Filardo G, Damiano RJ, Ailawadi G, et al. Epidemiology 
of new-onset atrial fibrillation following coronary artery bypass graft surgery. Heart 2018;104:985-92.

3. Filardo G, Ailawadi G, Pollock BD, et al. Postoperative atrial fibrillation: Sex-specific characteristics and effect on survival. J Thorac Cardiovasc Surg 2020;159:14191425.e1.

4. Axtell AL, Moonsamy P, Melnitchouk S, et al. Preoperative predictors of new-onset prolonged atrial fibrillation after surgical aortic valve replacement. J Thorac Cardiovasc Surg 2020;159:1407-14.

5. Ismail MF, El-Mahrouk AF, Hamouda TH, et al. Factors influencing postoperative atrial fibrillation in patients undergoing on-pump coronary artery bypass grafting, single center experience. J Cardiothorac Surg 2017;12:40.

6. Semsarian C, Ingles J, Maron MS, et al. New perspectives on the prevalence of hypertrophic cardiomyopathy. J Am Coll Cardiol 2015;65:1249-54.

7. Maron BJ, Maron MS. Hypertrophic cardiomyopathy. Lancet 2013;381:242-55.

8. Siontis KC, Geske JB, Ong K, et al. Atrial fibrillation in hypertrophic cardiomyopathy: prevalence, clinical correlations, and mortality in a large high-risk population. J Am Heart Assoc 2014;3:e001002.

9. Watanabe H, Watanabe T, Sasaki S, et al. Close bidirectional relationship between chronic kidney disease and atrial fibrillation: the Niigata preventive medicine study. Am Heart J 2009;158:629-36.

10. Massicotte-Azarniouch D, Kuwornu JP, Carrero JJ, et al. Incident Atrial Fibrillation and the Risk of Congestive Heart Failure, Myocardial Infarction, End-Stage Kidney Disease, and Mortality Among Patients with a Decreased Estimated GFR. Am J Kidney Dis 2018;71:191-9.

11. Soliman EZ, Prineas RJ, Go AS, et al. Chronic kidney disease and prevalent atrial fibrillation: the Chronic Renal Insufficiency Cohort (CRIC). Am Heart J 2010;159:1102-7.

12. Kornej J, Hindricks G, Banerjee A, et al. Changes in renal function after catheter ablation of atrial fibrillation are associated with CHADS2 and CHA2DS2-VASc scores and arrhythmia recurrences. Heart 2015;101:126-31.

13. Chua SK, Shyu KG, Lu MJ, et al. Renal dysfunction and the risk of postoperative atrial fibrillation after cardiac surgery: role beyond the CHA2DS2-VASc score. Europace 2015;17:1363-70.

14. Gersh BJ, Maron BJ, Bonow RO, et al. 2011 ACCF/ AHA guideline for the diagnosis and treatment of hypertrophic cardiomyopathy: a report of the American College of Cardiology Foundation/American Heart
Association Task Force on Practice Guidelines.

Circulation 2011;124:e783-831.

15. Elliott PM, Anastasakis A, Borger MA, et al. 2014 ESC Guidelines on diagnosis and management of hypertrophic cardiomyopathy: the Task Force for the Diagnosis and Management of Hypertrophic Cardiomyopathy of the European Society of Cardiology (ESC). Eur Heart J 2014;35:2733-79.

16. Jafar TH. Modified MDRD equations outperform the original equations in Chinese patients with chronic kidney disease. Nat Clin Pract Nephrol 2007;3:186-7.

17. Wang S, Cui H, Yu Q, et al. Excision of anomalous muscle bundles as an important addition to extended septal myectomy for treatment of left ventricular outflow tract obstruction. J Thorac Cardiovasc Surg 2016;152:461-8..

18. Kinoshita T, Asai T, Ishigaki T, et al. Preoperative heart rate variability predicts atrial fibrillation after coronary bypass grafting. Ann Thorac Surg 2011;91:1176-81.

19. Mack MJ, Pfister A, Bachand D, et al. Comparison of coronary bypass surgery with and without cardiopulmonary bypass in patients with multivessel disease. J Thorac Cardiovasc Surg 2004;127:167-73.

20. Tang B, Song Y, Cheng S, et al. In-Hospital Postoperative Atrial Fibrillation Indicates a Poorer Clinical Outcome after Myectomy for Obstructive Hypertrophic Cardiomyopathy. Ann Thorac Cardiovasc Surg 2020;26:22-29.

21. Rezaei Y, Peighambari MM, Naghshbandi S, et al. Postoperative Atrial Fibrillation Following Cardiac Surgery: From Pathogenesis to Potential Therapies. Am J Cardiovasc Drugs 2020;20:19-49.

22. Nguyen A, Schaff HV, Sedeek AF, et al. Septal Myectomy and Concomitant Coronary Artery Bypass Grafting for Patients with Hypertrophic Cardiomyopathy and Coronary Artery Disease. Mayo Clin Proc 2020;95:521-5.

23. Njoku A, Kannabhiran M, Arora R, et al. Left atrial volume predicts atrial fibrillation recurrence after radiofrequency ablation: a meta-analysis. Europace 2018;20:33-42.

24. Lee JS, Shim CY, Wi J, et al. Left ventricular diastolic function is closely associated with mechanical function of the left atrium in patients with paroxysmal atrial fibrillation. Circ J 2013;77:697-704.

25. Mita N, Kuroda M, Miyoshi S, et al. Association of Preoperative Right and Left Ventricular Diastolic Dysfunction with Postoperative Atrial Fibrillation in Patients Undergoing Lung Surgery: A Prospective Observational Study. J Cardiothorac Vasc Anesth 
2017;31:464-73.

26. Yamashita K, Hu N, Ranjan R, et al. Clinical Risk Factors for Postoperative Atrial Fibrillation among Patients after Cardiac Surgery. Thorac Cardiovasc Surg 2019;67:107-16.

27. Hashemzadeh K, Dehdilani M, Dehdilani M. Does Offpump Coronary Artery Bypass Reduce the Prevalence ofAtrial Fibrillation? J Cardiovasc Thorac Res 2013;5:45-9.

28. Athanasiou T, Aziz O, Mangoush O, et al. Does off-pump coronary artery bypass reduce the incidence of postoperative atrial fibrillation? A question revisited. Eur J Cardiothorac Surg 2004;26:701-10.

29. Almassi GH, Hawkins RB, Bishawi M, et al. New-onset postoperative atrial fibrillation impact on 5-year clinical outcomes and costs. J Thorac Cardiovasc Surg 2019. [Epub ahead of print]. doi: 10.1016/j.jtcvs.2019.10.150.

30. Bramer S, van Straten AH, Soliman HM, et al. The impact of new-onset postoperative atrial fibrillation on mortality after coronary artery bypass grafting. Ann Thorac Surg 2010;90:443-9.

31. Steinberg BA, Zhao Y, He X, et al. Management of postoperative atrial fibrillation and subsequent outcomes in contemporary patients undergoing cardiac surgery: insights from the Society of Thoracic Surgeons CAPS-Care Atrial Fibrillation Registry. Clin Cardiol 2014;37:7-13.

32. Yonezawa Y, Horinaka S, Shirakawa C, et al. Estimated glomerular filtration ratio is a better index than creatinine clearance (Cockcroft-Gault) for predicting the prevalence of atrial fibrillation in the general Japanese population. Hypertens Res 2018;41:451-9.

33. Franczyk B, Gluba-Brzozka A, Cialkowska-Rysz A, et al. The Problem of Atrial Fibrillation in Patients

Cite this article as: Meng Y, Wang S, Liu P, Zhang Y, Tang B, Zhu C, Wang S, Yang Q, Lu T, Nie C. The preoperative glomerular filtration rate predicts new-onset postoperative atrial fibrillation in patients with hypertrophic obstructive cardiomyopathy who undergo isolated septal myectomy. J Thorac Dis 2021;13(3):1612-1623. doi: 10.21037/jtd-20-3164 with Chronic Kidney Disease. Curr Vasc Pharmacol 2016;14:260-5.

34. Lau YC, Proietti M, Guiducci E, et al. Atrial Fibrillation and Thromboembolism in Patients with Chronic Kidney Disease. J Am Coll Cardiol 2016;68:1452-64.

35. Li M, Liu T, Luo D, et al. Systematic review and metaanalysis of chronic kidney disease as predictor of atrial fibrillation recurrence following catheter ablation. Cardiol J 2014;21:89-95.

36. Garg L, Gupta M, Sabzwari S, et al. Atrial fibrillation in hypertrophic cardiomyopathy: prevalence, clinical impact, and management. Heart Fail Rev 2019;24:189-97.

37. Vaidya K, Semsarian C, Chan KH. Atrial Fibrillation in Hypertrophic Cardiomyopathy. Heart Lung Circ 2017;26:975-82.

38. Huang CY, Yang YH, Lin LY, et al. Renin-angiotensinaldosterone blockade reduces atrial fibrillation in hypertrophic cardiomyopathy. Heart 2018;104:1276-83.

39. Prendergast HM, Dudley S, Brown M, et al. Antihypertensive medications and diastolic dysfunction progression in an African American population. High Blood Press Cardiovasc Prev 2014;21:269-74.

40. Guttmann OP, Pavlou M, O'Mahony C, et al. Prediction of thrombo-embolic risk in patients with hypertrophic cardiomyopathy (HCM Risk-CVA). Eur J Heart Fail 2015;17:837-45.

41. Li R, White CM, Mehmeti J, et al. Impact of a Perioperative Prophylaxis Guideline on PostCardiothoracic Surgery Atrial Fibrillation. Ann Pharmacother 2017;51:743-50. 